\title{
Joint hypermobility in adults referred to rheumatology clinics
}

\author{
Alan J Bridges, Elaine Smith, John Reid
}

\begin{abstract}
Joint hypermobility is a rarely recognised aetiology for focal or diffuse musculoskeletal symptoms. To assess the occurrence and importance of joint hypermobility in adult patients referred to a rheumatologist, we prospectively evaluated 130 consecutive new patients for joint hypermobility. Twenty women $(15 \%)$ had joint hypermobility at three or more locations ( $\geqslant 5$ points on a 9 point scale). Most patients with joint hypermobility had common musculoskeletal problems as the reason for referral. Two patients referred with a diagnosis of rheumatoid arthritis were correctly reassigned a diagnosis of hypermobility syndrome. Three patients with systemic lupus erythematosus had diffuse joint hypermobility. There was a statistically significant association between diffuse joint hypermobility and osteoarthritis. Most patients (65\%) had first degree family members with a history of joint hypermobility. These results show that joint hypermobility is common, familial, found in association with common rheumatic disorders, and statistically associated with osteoarthritis. The findings support the hypothesis that joint hypermobility predisposes to musculoskeletal disorders, especially osteoarthritis.
\end{abstract}

A commonly unrecognised aetiology for focal or diffuse musculoskeletal problems is the hypermobility syndrome. ${ }^{12}$ The hypermobility syndrome refers to a constellation of musculoskeletal symptoms and clinical findings of diffuse joint laxity in an otherwise normal subject. ${ }^{12}$ When not associated with musculoskeletal problems the clinical findings are best termed diffuse joint hypermobility. Although the clinical features may mimic inflammatory arthritis with joint swelling and pain, the course of joint hypermobility is not progressive, destructive, nor disabling. ${ }^{2-7}$

In population studies joint hypermobility has been found more often in children, with diminishing occurrence as age increases. ${ }^{89}$ Joint hypermobility was commonly noted in paediatric patients referred to a rheumatologist and in normal schoolchildren. ${ }^{4-6}$ Investigations in adults have suggested that joint hypermobility is associated with osteoarthritis, mitral valve prolapse, and chondrocalcinosis. ${ }^{10-13}$ Only one report has focused on the hypermobility syndrome as a diagnosis in adult patients referred to a rheumatologist. ${ }^{14}$ To assess the occurrence, modes of presentation, and associations of joint hypermobility in adult patients referred to a rheumatologist for musculoskeletal problems, we evaluated 130 consecutive new patients for joint hypermobility and associated clinical features.

\section{Patients and methods}

PATIENTS

One hundred and thirty consecutive adult patients (age $>18$ years) referred to the outpatient rheumatology clinic at the University of Missouri-Columbia for musculoskeletal problems or connective tissue disease were evaluated by ES and AJB. There were 97 women and 33 men with an average age of 51 years (range 18-83).

\section{METHODS}

A complete history and physical examination was performed including an examination for joint laxity. The criteria devised by Carter and Wilkinson ${ }^{15}$ with a modification by Beighton et $a l^{8}$ were used to assess hypermobility (table 1). A mobility score was calculated by allocating one point for the ability to perform the manoeuvre at each joint. Two points were allocated if hypermobility was detectable at bilateral joints. Truncal hypermobility received a score of 1 . A mobility score greater than or equal to 5 was considered consistent with joint hypermobility.

The following features were also assessed: $(a)$ easy bruising; $(b)$ history of joint dislocations; $(c)$ history of recurrent joint sprains; $(d)$ history or physical finding of joint swelling (effusion); $(e)$ loose skin or hernias; $(f)$ temporomandibular crepitus and pain; $(g)$ scoliosis; $(h)$ mitral valve prolapse by auscultation (click-murmur with characteristic changes on Valsalva manoeuvre) or echocardiography; $(i)$ passive hyperextension of one or more proximal interphalangeal joints to greater than $90^{\circ} ;(j)$ passive internal rotation of the hips greater than $45^{\circ} ;(k)$ passive dorsiflexion of the ankle beyond $15^{\circ}$; $(l)$ flat medial arch of the foot on standing.

The criteria used to make the diagnoses were

Table I Criteria used to evaluate joint hypermobility. Based on Carter and Wilkinson ${ }^{15}$ modified by Beighton et al

1 Passive dorsiflexion of fifth finger beyond $90^{\circ}$ at metacarpophalangeal joint

Passive apposition of thumb to flexor aspect of forearm Hyperextension of elbow beyond $10^{\circ}$

Hyperextension of knee beyond $10^{\circ}$

5 Forward flexion of the trunk with the knees fully extended so that the palms of the hands rest flat on the floor 
based on the accepted criteria of the American College of Rheumatology where applicable. ${ }^{16}$

Patients with findings including dislocation of the lens (or positive family history), aortic regurgitation or family history of aortic rupture, arachnodactyly, excessive arm span, marked skin hyperextensibility, or cigarette paper scars were suspected to have Marfan or Ehler-Danlos syndrome and not considered to have benign hypermobility syndrome.

\section{STATISTICS}

Categorical variables (two way tables) were tested using $\chi^{2}$ analysis. Continuous variables were tested using the Wilcoxon signed rank or Mann-Whitney U tests.

\section{Results}

Joint hypermobility was found in 20 (15\%) patients. Table 2 summarises the findings. The average age of the patients with joint hypermobility was 50 years (range 23-80), whereas patients without joint hypermobility had an average age of 52 years (range 18-83); $(\mathrm{p}>0.4)$. The patients with joint hypermobility had an average mobility score of 8 whereas patients without joint hypermobility had mobility scores of $\leqslant 2$ (average $0.4 ; p<0.001$ ). All patients with joint hypermobility were women whereas only $75 \%$ of the total number of referred patients were women. Most patients (65\%) with joint hypermobility reported a family history of joint hypermobility in at least one first degree relative. Hypermobility of the trunk was found in the younger patients with joint hypermobility (average age 37 years) but was not found in older patients with joint hypermobility (average age 62 years $)(p<0.005)$.

Table 2 shows that patients who met the criteria for joint hypermobility had other features of joint laxity. Many patients had hypermobility of the fingers (50\%), hips (35\%), and ankles $(50 \%)$. Eighty five per cent of the patients had flat feet and 35\% had scoliosis. Mitral valve prolapse was found in nine (45\%) patients. This was documented by echocardio- graphy in five patients. Easy bruising (three patients), temporomandibular joint dysfunction (two patients), history of joint effusions (five patients), and recurrent sprains (three patients), were rare. Joint dislocations, loose skin, and hernias were not noted in any patient.

A primary diagnosis of hypermobility syndrome was made in two young women with joint mobility scores of 9, recurrent joint effusions, scoliosis, and mitral valve prolapse. The two patients were referred with a diagnosis of rheumatoid arthritis. One patient also had temporomandibular joint dysfunction and chronic low back pain from sacroiliac hypermobility that became symptomatic during her first pregnancy. Neither patient had eye, aortic, skin, or other skeletal abnormalities suggestive of Marfan or Ehlers-Danlos syndrome.

Table 2 gives the primary diagnosis for the main symptom of each patient with joint hypermobility. Causes for the main symptom other than joint hypermobility were found in 18/20 patients. Osteoarthritis and fibromyalgia were commonly noted in the patients with joint hypermobility. When all diagnoses were taken into consideration, osteoarthritis was found in $12 / 20(60 \%)$ patients with joint hypermobility. In contrast, $33 / 110(30 \%)$ patients without joint hypermobility had osteoarthritis $\left(\chi^{2}=6 \cdot 73\right.$; $\mathrm{p}<0.01)$. The age of the patients with osteoarthritis and joint hypermobility did not differ significantly from the age of the patients with osteoarthritis without joint hypermobility (60 and 66 years respectively; $p>0.05$ ). Fibromyalgia was common, but its occurrence did not differ significantly between the patients with (30\%) and without joint hypermobility $(38 \%)(p>0 \cdot 05)$. No other musculoskeletal problem occurred more often in patients with joint hypermobility compared with patients without joint hypermobility.

Joint hypermobility was found in three patients with systemic lupus erythematosus (SLE) and one patient with incomplete lupus erythematosus. A patient with rheumatoid arthritis had a joint mobility score of 5 . This patient had a history of hypermobility of the hands and wrists before the onset of rheumatoid

Table 2 Characteristics of patients with joint hypermobility

\begin{tabular}{|c|c|c|c|c|c|c|c|c|c|c|c|c|c|c|}
\hline \multirow{2}{*}{$\begin{array}{l}\text { Patient } \\
\text { No }\end{array}$} & \multirow{2}{*}{$\begin{array}{l}\text { Age } \\
\text { (years) }\end{array}$} & \multicolumn{5}{|c|}{ Criteria for joint hypermobility } & \multicolumn{7}{|c|}{ Other features } & \multirow{2}{*}{$\begin{array}{l}\text { Diagnosis for } \\
\text { chief symptom }\end{array}$} \\
\hline & & $M C P^{*}$ & Thumb & Elbow & Knee & Back & $P I P^{*}$ & Swan & Hip & Ankle & Scoliosis & $M V P^{*}$ & Flat feet & \\
\hline 1 & 34 & + & + & + & + & + & + & + & - & + & - & - & + & FM \\
\hline 2 & 80 & + & + & + & + & - & - & - & - & - & - & - & + & $\mathrm{OA}$ \\
\hline 3 & 48 & + & + & + & + & - & - & - & - & - & - & - & - & SLE, FM \\
\hline 4 & 44 & + & + & + & + & - & - & - & - & + & + & - & - & FM \\
\hline 5 & 38 & + & + & + & + & + & - & - & - & + & + & - & - & Bursitis \\
\hline 6 & 73 & - & + & + & + & - & - & - & - & - & - & A & + & OA \\
\hline 7 & 23 & + & + & + & + & + & + & + & + & + & - & $\mathbf{E}$ & + & OA \\
\hline 8 & 54 & + & + & + & + & - & + & + & + & + & + & $\mathbf{E}$ & + & SLE, OA \\
\hline 9 & 76 & + & + & + & - & - & - & + & - & - & - & A & + & $\mathrm{OA}$ \\
\hline 10 & 58 & + & + & + & + & - & + & + & - & + & - & $=$ & + & OA \\
\hline 11 & 34 & + & + & + & + & + & + & + & + & + & + & $\mathbf{E}$ & + & OA \\
\hline 12 & 38 & + & + & + & + & + & + & - & + & - & + & A & + & ILE \\
\hline 13 & 48 & + & + & + & + & + & + & + & - & - & - & $\bar{x}$ & + & SLE, OA \\
\hline 14 & 49 & + & + & + & + & + & + & + & + & - & + & $\mathbf{E}$ & + & MFP \\
\hline 15 & 57 & + & + & + & + & - & + & + & - & + & - & - & + & FM, OA \\
\hline 16 & 72 & + & + & + & + & - & - & - & + & + & - & - & + & OA, chondrocalcinosis \\
\hline 17 & 59 & + & + & + & + & - & - & - & - & - & - & - & + & $\mathrm{OA}$, tendonitis \\
\hline 18 & 31 & + & + & + & + & + & + & + & - & + & + & A & + & Bursitis, MFP \\
\hline 19 & 34 & - & - & + & + & + & - & - & + & + & + & - & + & RA \\
\hline 20 & 41 & + & + & + & + & + & - & - & - & - & - & - & + & OA \\
\hline
\end{tabular}

${ }^{*}(+)$ finding present; $(-)$ finding not present; $\overline{(\overline{M C P})}$ metacarpal phalangeal joint; (PIP) proximal interphalangeal joint; (MVP) mitral valve prolapse; (A) auscultation (E) finding present; (-) finding not present; $($ COP) metacarpal phalangeal joic lupus erythematosus; (ILE) incomplete lupus erythematosus; (MFP) myofascial pain; (E) echocardiography; (FM) rheumatoid arthritis. 
arthritis. Each of the patients had intermittent arthralgia which may have been due to joint hypermobility. In two patients these symptoms had been treated with corticosteroids with partial response.

\section{Discussion}

The evaluation of 130 consecutive adults referred to a rheumatologist for musculoskeletal problems showed that joint hypermobility was common, familial, found in association with musculoskeletal disorders, and statistically associated with osteoarthritis.

Previous studies of joint mobility have shown that mobility decreases with age with a normal score in the adult of $0-2 .^{18}$ Joint mobility scores of $\geqslant 5$ were found in less than $2 \%$ of randomly selected adults. ${ }^{8}$ Other studies performed in selected populations (musicians and gymnasts) showed joint hypermobility to be more common, but unfortunately details of age relationships were not given. ${ }^{17} 18$ In this study, joint hypermobility was fairly common (15\%) and the patients had a mean age of 50 years. The patients did not have common occupations, hobbies, or skills. These comparisons suggest that joint hypermobility in patients with musculoskeletal problems severe enough to be referred to a rheumatologist is more common than in the general population. Optimally, an age matched control population should be evaluated for comparison.

Grahame reported a diagnostic survey of referrals to a large rheumatology clinic showing that hypermobility syndrome was diagnosed in $3 \cdot 25 \%$ of women. ${ }^{14}$ This corresponds closely with the $2.06 \%$ of our study population of women (two of 97) with hypermobility syndrome.

Laxity of the joints has been suggested as a predisposing factor for joint or soft tissue injury. 12519 This may account for the high percentage of subjects $(15 \%)$ in this study with joint hypermobility among patients referred to a rheumatologist. Osteoarthritis was particularly common in the patients with joint hypermobility in this study, consistent with other reports. ${ }^{1011}$ Although a direct association between joint hypermobility, joint or soft tissue injury, and osteoarthritis cannot be made from this study, this relationship warrants further evaluation. The aetiology and pathogenesis of osteoarthritis is unknown but may be associated with abnormal joint connective tissue and abnormal joint biomechanics of stress. ${ }^{13}$ Hypermobile joints may be associated with abnormal 'loose' connective tissue and abnormal joint biomechanics. ${ }^{212}{ }^{13}$ Thus joint hypermobility may be associated with two mechanisms which are suggested as playing a causative part in the pathogenesis of osteoarthritis.

Clinical features of joint hypermobility may mimic systemic rheumatic disease. ${ }^{46}$ This study supports this concept. Two patients presented with a diagnosis of rheumatoid arthritis, although further evaluation substantiated a diagnosis of hypermobility syndrome. This study also emphasises the importance of distinguishing joint hypermobility from coexisting, well defined systemic rheumatic diseases.
Five patients with systemic rheumatic diseases had arthralgias which may have been due to joint hypermobility and may have been inappropriately treated. Improved recognition of joint hypermobility could cirumvent these problems.

The finding of joint hypermobility in three patients with SLE and one with incomplete lupus erythematosus may represent a new clinical association. Babini et al recently reported that $10 / 59(17 \%)$ patients with SLE had joint hypermobility. ${ }^{20}$ They suggested that joint hypermobility may be related to ligamentous laxity and instability in association with inflammatory arthritis or hyperparathyroidism secondary to chronic renal failure. None of the lupus patients in this study had acute or chronic synovitis or renal insufficiency, and each patient had a history of hypermobile features since childhood (data not shown). The hereditary nature of hypermobility suggested by this study and others ${ }^{3} 2122$ may be linked to the genetic associations of SLE. The association of joint hypermobility and SLE, however, is more likely to be related to the occurrence of two entities which are common in young women.

This study lends further support to the association of mitral valve prolapse and joint hypermobility. ${ }^{122-25}$ Mitral valve prolapse was the most common extra-articular finding in patients with joint hypermobility. This association strengthens the hypothesis that patients with diffuse joint hypermobility have a generalised connective tissue abnormality.

In contrast with other reports which emphasise the occurrence of hypermobility in younger patients, we found hypermobile joints in four elderly patients. ${ }^{89}$ No patient in this study had features of Marfan or Ehlers-Danlos syndrome; however, four patients maintained hypermobile joints to the ages of $72,73,76$, and 80 years. Joint hypermobility must be considered in the differential diagnosis of musculoskeletal symptoms in patients of all ages.

In conclusion, we found joint hypermobility in $15 \%$ of adult patients referred to a rheumatologist. It is important for doctors to recognise this problem to ensure correct diagnosis and treatment because joint hypermobility may mimic rheumatic diseases. Further evaluation of the association between joint hypermobility and diseases such as osteoarthritis and SLE is warranted.

We appreciate the thoughtful review of the manuscript by Drs Sara Walker and James Cassidy. This work was supported in part
by NIDRR, Research and Training Center Grant, H133B80075.

1 Kirk J A, Ansell B M, Bywaters E G L. The hypermobility syndrome: musculoskeletal complaints associated with generalized joint hypermobility. Ann Rheum Dis 1967; 26 . $419-25$

2 Grahame R. The hypermobility syndrome. Ann Rheum Dis 1990; 49: 199-200.

3 Child A H. Joint hypermobility syndrome: inherited disorde of collagen synthesis. $\mathcal{F}$ Rheumatol 1986; 13: 239-42

4 Gedalia A, Person D A, Brewer E J Jr, Giannini E Hypermobility of the joints in juvenile episodic arthritis/ arthralgia. F Pediatr 1985; 107: 873-6.

5 Lewkonia $R$ M, Ansell B M Articular hypermobility imulating chronic rheumatic disease. Arch Dis Child 1983; 58: 988-92.

6 Biro F, Gewanter H L, Baum J. The hypermobility syndrome. Pediatrics 1983; 72: 701-6.

7 Jessee E F, Owen D S Jr, Sagar K B. The benign hypermobile joint syndrome. Arthritis Rheum 1980; 23: $1053-6$. 
8 Beighton P, Solomon L, Soskolne C L. Articular mobility in an African population. Ann Rheum Dis 1973; 32: 413-8. 9 Wynne-Davies R. Familial joint laxity. Proc R Soc Med 1971, 64: 689-90.

10 Scott $\mathrm{D}$, Bird $\mathrm{H}$, Wright $\mathrm{V}$. Joint laxity leading to osteoarthrosis. Rheumatol Rehabil 1979; 18: 167-9.

11 Bird H A, Tribe C R, Bacon P A. Joint hypermobility leading to osteoarthrosis and chondrocalcinosis. Ann Rheum Dis 1978; 37: 203-11.

12 Handler C E, Child A, Light N D, Dorrance D E. Mitral valve prolapse, aortic compliance, and skin collagen in join hypermobility syndrome. Br Heart $\mathcal{f}$ 1985; 54: 501-8.

13 Grahame R. How often, when and how does joint hyper mobility lead to osteoarthritis? $\mathrm{Br} \mathcal{J}$ Rheumatol 1989; 28 320.

14 Grahame R. Clinical manifestations of the joint hypermobility syndrome. Reumatologia (USSR) 1986; 2: 20-4.

15 Carter C, Wilkinson J. Persistent joint laxity and congenita dislocation of the hip. 7 Bone foint Surg $[B r] 1964 ; 46$; $40-5$.

16 Schumacher H R, ed. Primer on the rheumatic diseases. 1988. Beighton P B, Grahame R, Bird H A. Hypermobility of joints. 2nd ed. Berlin: Springer, 1989.

18 Larsson L-G, Baum J, Mudholkar G S. Hypermobility: teatures and difterential incidence between the sexes. Arthritis Rheum 1987; 30: 1426-30.

19 Nicholas J A. Injuries to knee ligaments: relationship to looseness and tightness in football players. $7 A M A 1970$ 212: 2236-9.

20 Babini S M, Cocco J A M, Babini J C, de la Sota M, Arturi A Marcos J C. Atlantoaxial subluxation in systemic lupus erythematosus: further evidence of tendinous alterations. F Rheumatol 1990; 17: 173-7.

21 Beighton $\mathrm{P} \mathrm{H}$, Horan $\mathrm{F}$ T. Dominant inheritance in familia generalized articular hypermobility. F Bone foint Surg [Br] 1970; 52: 145-7.

22 Horan F T, Beighton P H. Recessive inheritance of generalized joint hypermobility. Rheumatol Rehabil 1973; 12: 47-9.

23 Grahame R, Edwards J C, Pitcher D, Gabell A, Harvey W. A clinical and echocardiographic study of patients with th hypermobility syndrome. Ann Rheum Dis 1981; 40: 541-6.

24 Pitcher D, Grahame R. Mitral valve prolapse and join hypermobility: evidence for a systemic connective tissue abnormality? Ann Rheum Dis 1982; 41: 352-4.

25 Ondrasik M, Rybar I, Rus V, Bosak V. Joint hypermobility in primary mitral valve prolapse patients. Clin Rheumato in primary mitra

I 